\title{
An irrational problem
}

\author{
by \\ Franklin D. Tall (Toronto)
}

\begin{abstract}
Given a topological space $\langle X, \mathcal{T}\rangle \in M$, an elementary submodel of set theory, we define $X_{M}$ to be $X \cap M$ with topology generated by $\{U \cap M: U \in \mathcal{T} \cap M\}$. Suppose $X_{M}$ is homeomorphic to the irrationals; must $X=X_{M}$ ? We have partial results. We also answer a question of Gruenhage by showing that if $X_{M}$ is homeomorphic to the "Long Cantor Set", then $X=X_{M}$.
\end{abstract}

In [JT], we considered the elementary submodel topology, defined as follows. Let $\langle X, \mathcal{T}\rangle$ be a topological space which is an element of $M$, an elementary submodel of the universe of sets. (Actually, an elementary submodel of $H(\theta)$ for $\theta$ a sufficiently large regular cardinal. See [KT] or Chapter 24 of $[\mathrm{JW}]$ for discussion of this technical point.) Let $\mathcal{T}_{M}$ be the topology on $X \cap M$ generated by $\{U \cap M: U \in \mathcal{T} \cap M\}$. Then $X_{M}$ is defined to be $X \cap M$ with topology $\mathcal{T}_{M}$. In $[\mathrm{T}]$ we raised the question of recovering $X$ from $X_{M}$ and proved for example:

THEOREM 1. If $X_{M}$ is a locally compact uncountable separable metrizable space, then $X=X_{M}$.

In particular:

Corollary 2. If $X_{M}$ is homeomorphic to $\mathbb{R}$, then $X=X_{M}$.

The proof proceeded by showing that $[0,1] \subseteq M$-any definable set of power $2^{\aleph_{0}}$ would do. It followed that $\omega_{1} \subseteq M$, whence by relativization we deduced that $X$ had no uncountable left- or right-separated subspaces. This implied both $X$ and $\mathcal{T}$ had cardinality $\leq 2^{\aleph_{0}}$ and hence were included in $M$. This sort of proof will recur frequently in what follows.

2000 Mathematics Subject Classification: Primary 03C62, 03E35, 54A35, 54B99; Secondary 03E15, 03E55, 54F65, 54G20, 54H05.

Key words and phrases: elementary submodel, irrationals, reals, Cantor Set, Bernstein Set, Long Cantor Set.

Research partially supported by NSERC Grant A-7354. 
On the other hand, we noted in $[\mathrm{JT}]$ :

ExAMPLE. Let $\langle X, \mathcal{T}\rangle$ be any regular space without isolated points, $X, \mathcal{T} \in M$, a countable elementary submodel. Then $X_{M}$ is homeomorphic to $\mathbb{Q}$.

The problem that naturally arises then is:

Problem. If $X_{M}$ is homeomorphic to $\mathbb{R}-\mathbb{Q}$, is $X$ also? In particular, does $X=X_{M}$ ?

This is the problem of our title.

Undefined topological terms can be found in [E], set-theoretic ones in $[\mathrm{Ku}]$, with the exception of $0^{\#}$, Jónsson cardinal, and Chang's Conjecture, for which consult $[K]$. However let us define two topological notions which may not be familiar to set theorists:

Definition. A space is of pointwise countable type if each point is contained in a compact set $K$ for which there exist open $\left\{U_{n}\right\}_{n<\omega}$ including $K$ such that each open set including $K$ includes some $U_{n}$. A map is perfect if it is continuous, closed, and pre-images of points are compact.

We obtained a partial result concerning the Irrational Problem in $[\mathrm{T}]$ :

THEOREM 3. Suppose $0^{\#}$ does not exist and $X_{M}$ is a separable metrizable space of cardinality continuum (e.g. an uncountable separable completely metrizable space). Then $X=X_{M}$.

The only use of the set-theoretic hypothesis (which follows for example from $V=L$ ) was to conclude (via $[\mathrm{KT}]$ ) that $2^{\aleph_{0}} \subseteq M$ since $|M| \geq 2^{\aleph_{0}}$. As noted by Welch [W], this also follows from the assumption that $2^{\aleph_{0}}$ is not a Jónsson cardinal. Once one has $2^{\aleph_{0}} \subseteq M$, the proof outlined above for Theorem 1 applies in this situation. In this paper we obtain several more partial results:

TheOREM 4. Suppose $X_{M}$ is an uncountable separable completely metrizable space. Then $X=X_{M}$ if any of the following conditions hold:

(a) $X$ includes a perfect pre-image of a compact first countable uncountable $T_{2}$ space,

(b) $X$ is (a product of spaces) of pointwise countable type, e.g. $X$ is first countable or locally compact or Čech-complete,

(c) $|\mathbb{R} \cap M|$ is uncountable,

(d) $|X|=2^{\aleph_{0}}$.

For all I know, the theorem is true without any additional hypothesis, at least for $\mathbb{R}-\mathbb{Q}$. Chang's Conjecture, which yields an uncountable $M$ with $M \cap \omega_{1}$ countable, is a natural hypothesis to use, say with $\mathrm{CH}$, to try to get a counterexample. (This conjunction does yield a non-metrizable $X$ such 
that $X_{M}$ is a separable metrizable space of size $2^{\aleph_{0}}[\mathrm{~T}]$.) Note that (a) and (b) rule out a number of plausible attempts, such as the "Irrational Long Line" or the product of $\aleph_{1}$ copies of the countable discrete space.

We will prove (a) and (b) first. Their proofs are natural extensions of the proof of Theorem 1. (b) in fact follows immediately from (a) by using Lemmas 5 and 6 below together with the classical topological fact that an uncountable separable completely metrizable space includes a copy of the Cantor Set.

Lemma 5 [JT]. If $X_{M}$ is $T_{i}, i \leq 3$, so is $X$.

Lemma 6. If $X$ is $T_{3}$ and a product of spaces of pointwise countable type, then $X_{M}$ is a perfect image of a subspace of $X[\mathrm{P}]$. For $X$ compact $T_{2}$, $X_{M}$ is a perfect image of $X$ itself [Ju].

(A proof of Lemma 6 for spaces of pointwise countable type may be found in [JT]. Alternatively, one can omit Lemma 6 and divide into 2 cases: finite products of spaces of pointwise countable type have pointwise countable type, so [JT] applies; infinite products include copies of the Cantor Set.)

The idea of the proof of (a) is to use the perfect map plus another classical fact, namely that the Cantor Set maps onto $[0,1]$, to obtain via elementarity a closed map from a closed-hence complete - subspace of $X_{M}$ onto $[0,1]_{M}=[0,1] \cap M$. (The equality is by first countability [JT].) Closed metrizable images of completely metrizable spaces are completely metrizable (see e.g. [E, 4.4.17]), hence absolute $G_{\delta}$. If $[0,1] \cap M$ is a $G_{\delta}$ in $[0,1]$, it is in fact all of $[0,1]$ (see below), so $[0,1] \subseteq M$, which, by the proof of Theorem 1 , is enough to get the desired result. Unfortunately, elementarity does not quite give us a closed map, so we have to work a bit harder. Now for the details. We first need definitions and a technical lemma which enable us to make do with an elementary version of a closed map.

Definition. A regular space is almost Čech-complete if there is a sequence $\{\mathcal{G}(n)\}_{n<\omega}$, each $\mathcal{G}(n)$ a collection of open sets such that $\bigcup \mathcal{G}(n)$ is dense (we call $\mathcal{G}(n)$ an "open almost-cover"), and such that whenever $\mathcal{F}$ is a centered collection of open sets meeting each $\mathcal{G}(n)$, then $\bigcap\{\bar{F}: F \in \mathcal{F}\} \neq 0$. If we require the $\mathcal{G}(n)$ 's to be covers, we get Čech-completeness.

Lemma $7[\mathrm{~F}]$. A completely regular space is almost Čech-complete if and only if it includes a dense $G_{\delta}$ Čech-complete subspace.

Definition. Let $\mathcal{B}$ be a basis for a space $X$ such that $\mathcal{B}$ is closed under finite intersections. $f: X \rightarrow Y$ is a $\mathcal{B}$-map if:

(1) for all $B \in \mathcal{B}, f(X-B)$ is closed,

(2) $f$ is onto, but for no $B \in \mathcal{B}$ is $f \mid(X-B)$ onto $Y$. 
Lemma 8. A $\mathcal{B}$-image of an almost $\check{C}$ ech-complete space is almost $\check{C} e c h$ complete.

Proof. We model our proof on that for closed irreducible images in [AL]. Suppose $X$ is almost Čech-complete with respect to the sequence $\{\mathcal{G}(n)\}_{n<\omega}$ of open almost-covers. Let $\mathcal{B}$ be a basis for $X$. For each $n<\omega$ and each $x \in \bigcup \mathcal{G}_{n}$, pick a basic open $G_{n}^{\prime}(x) \in \mathcal{B}$ containing $x$ and included in some member of $\mathcal{G}(n)$. Then, letting $\mathcal{G}^{\prime}(n)=\left\{G_{n}^{\prime}(x): x \in \bigcup \mathcal{G}_{n}\right\}$, claim $X$ is almost Cech-complete with respect to the sequence $\left\{\mathcal{G}^{\prime}(n)\right\}_{n<\omega}$. For let $\mathcal{D}$ be a centered collection of open sets meeting each $\mathcal{G}^{\prime}(n)$, say $D_{n} \in \mathcal{D} \cap \mathcal{G}_{n}^{\prime}$. Then $D_{n}$ is included in some $D_{n}^{*} \in \mathcal{G}_{n}$ by construction. Then $\mathcal{D}^{*}=\mathcal{D} \cup\left\{D_{n}^{*}\right\}_{n<\omega}$ is centered, so $\bigcap\left\{\bar{D}: D \in \mathcal{D}^{*}\right\} \neq 0$, so $\bigcap\{\bar{D}: D \in \mathcal{D}\} \neq 0$.

So without loss of generality, we may assume each $\mathcal{G}_{n}$ is included in the basis $\mathcal{B}$. Given $B \in \mathcal{B}$, let $\widehat{B}=f^{-1}(Y-f(X-B))$. Then $\widehat{B}$ is open and included in $B$. Furthermore, by (2) (irreducibility), $\widehat{B} \neq \emptyset$. Indeed, $\widehat{B}$ is dense in $B$. To see this, let $U \in \mathcal{B}$ be such that $U \cap B \neq \emptyset$. Then $\emptyset \neq \widehat{U \cap B} \subseteq U \cap B$, but also $\widehat{U \cap B} \subseteq \widehat{B}$, since $x \in \widehat{U \cap B}$ implies $f(x) \in$ $Y-f(X-(U \cap B))$ implies $f(x) \notin f(X-B)$ implies $f(x) \in Y-f(X-B)$ implies $x \in f^{-1}(Y-f(X-B))$. Thus $\widehat{B} \cap U \neq \emptyset$.

Let $\mathcal{H}(n)=\{f(\widehat{G}): G \in \mathcal{G}(n)\}$. Claim $\mathcal{H}(n)$ is an open almost-cover of $Y$. Certainly each $f(\widehat{G})$ is open; given $U$ open in $Y$, some $G \in \mathcal{G}(n)$ meets $f^{-1}(U)$, hence so does $\widehat{G}$, hence $f(\widehat{G})$ meets $f^{-1}(U)$, hence so does $\widehat{G}$, hence $f(\widehat{G})$ meets $U$. Now suppose $\mathcal{D}$ is a centered family of open subsets of $Y$ meeting each $\mathcal{H}(n)$. Then $\mathcal{C}=\left\{f^{-1}(D): D \in \mathcal{D}\right\}$ is a centered family of open subsets of $X$ meeting each $\mathcal{G}(n)$, so $\bigcap\{\bar{C}: C \in \mathcal{C}\} \neq 0$. If $x \in \bigcap\{\bar{C}: C \in \mathcal{C}\}$, then $f(x) \in \bigcap\{\bar{D}: D \in \mathcal{D}\}$. So $Y$ is almost Cech-complete with respect to $\{\mathcal{H}(n)\}_{n<\omega}$.

Continuing with the proof of Theorem 4(a), with the aid of Lemmas 7 and 8 we can prove:

Lemma 9. Suppose $X$ includes a perfect pre-image of an uncountable compact first countable $T_{2}$ space $Y$ and $X_{M}$ is Čech-complete. Then $M \supseteq$ $[0,1]$.

Proof. First of all, as in the classical proof that uncountable compact first countable $T_{2}$ spaces have cardinality $2^{\aleph_{0}}$ (see e.g. [H, 4.5]), we notice that each such space $Y$ includes a compact subspace without isolated points, and hence a Cantor tree of closed sets, which yields a map of the subspace onto the Cantor Set (see e.g. $\left[\mathrm{J}_{2}, 3.18\right]$ ) and hence onto $[0,1]$. By the Tietze Extension Theorem, this map extends over the whole compact space $Y$ to give us a perfect map of it onto $[0,1]$. Since the composition of perfect maps is perfect, the perfect pre-image of a compact space is compact, and perfect maps from a compact space are closed irreducible on a closed subspace, we 
conclude that there is a closed $L \subseteq X$ and a closed irreducible map from $L$ onto $[0,1]$. By elementarity, there is an $F \in M$ such that:

(1) $F \cap M$ is closed in $X_{M}$,

(2) there is a continuous surjection $g$ from $F \cap M$ (as a subspace of $X_{M}$ ) to $[0,1] \cap M$ such that if $H \in M$ is a closed subset of $X$, then

(a) $g(F \cap H \cap M)$ is closed in $[0,1] \cap M$,

(b) if $g(F \cap H \cap M)=[0,1] \cap M$, then $F \cap H \cap M=F \cap M$.

We thus have a $\mathcal{B}$-map from a closed (hence Cech-complete) subspace of $X_{M}$ onto $[0,1] \cap M$. Therefore $[0,1] \cap M$ includes a dense Cech-complete subspace. Such a subspace is then $G_{\delta}$ in $[0,1] \cap M$. It follows that $(0,1) \cap M$ also includes a dense $G_{\delta}$. But $(0,1) \cap M$ is a topological subgroup of $(0,1)$ and we have shown it is non-meager. It has the property of Baire since $(0,1)-[(0,1) \cap M]$ is meager. We now apply:

Lemma 10 [Ke, 0.11]. A subgroup of a topological group which is nonmeager and has the property of Baire is clopen.

But $(0,1)$ is connected, so $(0,1) \cap M=(0,1)$, so $M$ includes $(0,1)$ and hence $[0,1]$ and we have established Lemma 9.

Theorem 4(a) can now easily be established in the manner of Theorem 1. Precisely:

Lemma 11. If $[0,1] \subseteq M$ and $X_{M}$ is $T_{2}$, hereditarily Lindelöf and hereditarily separable, then $X=X_{M}$.

The proof is outlined following Corollary 2 and is given in full in [T].

Theorem 4(c) is proved in a completely different fashion from 4(a), using the following unpublished result of $\mathrm{W}$. H. Woodin, included with his kind permission.

TheOREM 12. If $\mathbb{R} \cap M$ includes a perfect set, then $\mathbb{R} \cap M=\mathbb{R}$.

The following proof, due to E. T. Eisworth and included with his kind permission, is much more elementary than Woodin's proof.

Proof of Theorem 12. By a routine modification of the construction of a Bernstein set, construct $2^{\aleph_{0}}$ disjoint Bernstein sets of $\mathbb{R}$, i.e. $\left\{B_{r}\right\}_{r \in \mathbb{R}}$ such that neither $B_{r}$ nor $\mathbb{R}-B_{r}$ includes a Cantor Set. Define $f: \mathbb{R} \rightarrow \mathbb{R}$ by sending $B_{r}$ to $r, f$ arbitrary outside $\bigcup_{r \in \mathbb{R}} B_{r}$. Then there are such Bernstein sets and such an $f \in M$. If $P \subseteq \mathbb{R} \cap M=\mathbb{R}_{M}$ is perfect, it includes a Cantor Set and so meets each $B_{r}$. Therefore $f^{\prime \prime} P=\mathbb{R} \subseteq M$.

It follows immediately that:

Corollary 13. If $\mathbb{R} \cap M$ is analytic and uncountable, then $\mathbb{R} \cap M=\mathbb{R}$. 
Thus, to prove (c) we need only show $\mathbb{R} \cap M$ is analytic, which follows from the following two lemmas. We first quote:

LeMma 14 [E, 4.3.20]. If $Y$ is a completely metrizable space, then every continuous map $f: A \rightarrow Y$ from a dense subset $A$ of a topological space $X$ to the space $Y$ is extendible to a continuous map $F: B \rightarrow Y$ defined on a $G_{\delta}$ set $B \subseteq X$ including $A$.

We also need:

Lemma 15 [E, Exercise 6.2.A.(e)]. Every uncountable separable completely metrizable space without isolated points includes a dense subspace homeomorphic to $\mathbb{R}-\mathbb{Q}$.

Since the perfect kernel of a separable completely metrizable space is completely metrizable (as only countably many points have been removed and a $G_{\delta}$ subspace of a complete metric space is completely metrizable), we see that every uncountable separable completely metrizable space $X$ has the form $Y \cup I$, where $Y$ has a dense $G_{\delta}$ subspace homeomorphic to $\mathbb{R}-\mathbb{Q}\left(G_{\delta}\right.$ since $\mathbb{R}-\mathbb{Q}$ is completely metrizable) and $I$ is a countable set of isolated points. Applying this to $X_{M}$, let $L \subseteq X_{M}$ be homeomorphic to $\mathbb{R}-\mathbb{Q}$ and dense in the non-isolated part of $X_{M}$, say $h: L \cong \mathbb{R}-\mathbb{Q}$. Then $L$ is $G_{\delta}$ in $X_{M}$. Apply Lemma 14 now, taking $X \cap M$ for $A, \overline{X \cap M}$ for $X, X_{M}$ for $Y$ and the identity function for $f$. Since $f$ is onto, so will be $F$. Then $F^{-1}(L)$ is $G_{\delta}$ in a $G_{\delta}$ subset of a closed subset of $X$, and $h \circ F$ maps $F^{-1}(L)$ onto $\mathbb{R}-\mathbb{Q}$. Relativizing to $M$, we see that $(\mathbb{R}-\mathbb{Q}) \cap M$ is a continuous image of a $G_{\delta}$ subset of $X_{M}$ and hence is analytic. Then so is $\mathbb{R} \cap M$, and we are done.

Theorem 4(d) follows quickly from (c), for if $|X|=2^{\aleph_{0}}$, then $|X \cap M|=$ $\left|2^{\aleph_{0}} \cap M\right|$. But $|X \cap M|=2^{\aleph_{0}}$, so $2^{\aleph_{0}} \cap M$ is uncountable.

$|X|=2^{\aleph_{0}}$ will occur if $X$ is hereditarily Lindelöf; this is interesting because there is a consistent example - due to G. Gruenhage - of an $X$ which is hereditarily separable, hereditarily Lindelöf, non-metrizable, but for which $X_{M}$ is a separable metrizable space [Ju]. Such an example thus cannot have $X_{M}$ complete.

We will also use Lemma 9 to answer a question of Gruenhage. In [PT], we proved:

Theorem 16. (a) If $\omega_{1} \subseteq M$ and $X_{M}$ is homeomorphic to $\omega_{1}$, then $X=X_{M}$.

(b) Chang's Conjecture implies there is an $M$ such that $\left(\omega_{2}\right)_{M}$ is homeomorphic to $\omega_{1}$.

(c) If $X_{M}$ is homeomorphic to the Long Line, then $X=X_{M}$. (More generally, if $X_{M}$ is locally compact $T_{2}$, locally hereditarily Lindelöf, and connected, then $X=X_{M}$.) 
The proof of (c) used connectedness in an essential way, so Gruenhage (personal communication) asked what happens if we for example have $X_{M}$ homeomorphic to the "Long Cantor Set". We can answer that $X=X_{M}$ in that case and indeed for more general ones.

Let us call a line space the linearly ordered topological space obtained by replacing each $\alpha \in \omega_{1}$ by some non-empty compact subspace $\mathbb{R}_{\alpha}$ of $\mathbb{R}$. That is, we are considering the order (and resulting ordered topological space) on $\left\{\langle\alpha, r\rangle: \alpha \in \omega_{1}\right.$ and $\left.r \in \mathbb{R}_{\alpha}\right\}$ given by:

$$
\langle\alpha, r\rangle<\langle\beta, s\rangle \quad \text { if } \text { either } \alpha<\beta \text {, or } \alpha=\beta \text { and } r<s .
$$

The Long Cantor Set is obtained by taking each $\mathbb{R}_{\alpha}$ to be a copy of the Cantor Set $\mathbb{K}$.

We shall show:

THEOREM 17. If $X_{M}$ is homeomorphic to a line space, and some point of $X_{M}$ has no countable neighborhood, then $X=X_{M}$.

Corollary 18. If $X_{M}$ is homeomorphic to the Long Cantor Set, then $X=X_{M}$.

Rather than proving these directly (which can be done by an extension of the argument for Theorem 16(a), we shall obtain them as corollaries to more general results that - as in 16(c) - remove linearity from the picture. Before doing so, however, we offer up the following examples which show that finding the "right" generalization is not so easy.

THEOREM 19. (a) $2^{\aleph_{0}}<\aleph_{\omega}$ implies there is a locally compact $T_{2}$, locally hereditarily Lindelöf, countably compact space $X$ and an elementary submodel $M$ such that $X_{M} \neq X$, although $X_{M}$ is locally compact, $T_{2}$, locally hereditarily Lindelöf, countably compact, includes a Cantor Set, and has size $2^{\aleph_{0}}$.

(b) If $2^{\aleph_{0}}=\aleph_{1}$, we may additionally require that $X_{M}$ is the union of an increasing collection of $\aleph_{1}$ compact sets.

Proof. [JNW] construct locally compact $T_{2}$, locally countable, countably compact spaces $X_{n}$ of size $\aleph_{n}$, each $n \in \omega$. Suppose $2^{\aleph_{0}}=\aleph_{n}$; let $X$ be the disjoint sum of $[0,1]$ with $X_{n+1}$. Take a countably closed elementary submodel $M$ of size $2^{\aleph_{0}}=\aleph_{n}$ containing $X$. Then $X_{M} \neq X$, but includes $[0,1]$. By countable closure, $X_{M}$ is a countably compact subspace of the first countable space $X$, and hence is a closed subspace and therefore is locally compact. It also is locally hereditarily Lindelöf.

To prove (b), consider the space $X_{2}$ mentioned in the proof of (a). In fact - see $[\mathrm{JNW}]$ - this space has the property that countable sets have compact closure. Let $X=X_{2} \times[0,1]$. Let $M$ be a countably closed elementary submodel $M$ of size $2^{\aleph_{0}}$ containing $X_{2}$. Then $X_{M} \neq X$, but $X_{M}$ is locally 
compact $T_{2}$, locally hereditarily Lindelöf, has no point with a countable neighborhood, and countable sets have compact closure (since $X_{M}$ is a closed subspace of $X)$. Let $X_{M}=\left\{x_{\alpha}: \alpha<\omega_{1}\right\}$. Then $X_{M}=\bigcup_{\beta<\omega_{1}} \overline{\left\{x_{\alpha}: \alpha<\beta\right\}}$.

Despite these examples, there are non-linear generalizations of Theorem 17 available. First recall:

Definition. Let $\sigma$ be an ordinal. $\left\{x_{\alpha}\right\}_{\alpha<\sigma}$ is a free sequence (of points in a space $X)$ if for every $\beta<\sigma, \overline{\left\{x_{\alpha}: \alpha<\beta\right\}} \cap \overline{\left\{x_{\alpha}: \beta \leq \sigma\right\}}=0$.

We shall prove:

TheOREM 20. Suppose $X_{M}$ is locally compact $T_{2}$, locally hereditarily Lindelöf, contains a point with no countable neighborhood, and has no free sequence of length $>\mathfrak{c}^{2}$. Then $X_{M}=X$.

Arkhangel'skiı $[\mathrm{A}]$ proved that compact first countable spaces have no uncountable free sequences, so line spaces cannot have free sequences of length greater than $\omega_{1}^{2}$, so Theorem 17 and Corollary 18 immediately follow. After proving Theorem 20, we shall deduce some more corollaries.

We need to quote some results from previous papers that we will need in order to prove Theorem 20.

Lemma 21 [PT]. $X_{M}$ locally hereditarily Lindelöf and $\omega_{1} \subseteq M$ imply $X$ is locally hereditarily Lindelöf.

Lemma $22[\mathrm{~T}] . X_{M}$ locally compact $T_{2}$ implies $X$ is locally compact $T_{2}$.

Lemma 23 [JT]. $X$ first countable implies $X_{M}$ is a subspace of $X$.

Proof of Theorem 20. Since $X_{M}$ is locally compact $T_{2}$, by Lemma 6 some subspace of $X$ maps perfectly onto $X_{M}$ and hence $X$ includes a perfect pre-image of an uncountable compact first countable $T_{2}$ space. Thus by Lemma $9, M \supseteq[0,1]$ and hence $\omega_{1}$. Therefore $X$ is locally hereditarily Lindelöf and locally compact $T_{2}$ so it is first countable, so $X_{M}$ is a subspace of it.

In order to show that $X=X_{M}$, it will suffice to show that $|X| \leq 2^{\aleph_{0}}$. By local compactness, fix for each $x \in X$ an open set $U_{x}$ such that $\bar{U}_{x}$ is compact. Define a strictly increasing collection of closed subsets of $X$ as follows. Let $F_{0}$ be some $\bar{U}_{x_{0}}$. Given $F_{\alpha} \neq X$, take $y \in X-F_{\alpha}$ and let $F_{\alpha+1}=\overline{\bigcup\left\{U_{x}: x \in F_{\alpha}\right\} \cup U_{y}}$. For $\alpha$ limit with $\operatorname{cf}(\alpha)=\omega$, if $\overline{\bigcup_{\beta<\alpha} F_{\beta}} \neq X$, take $y \in X-\overline{\bigcup_{\beta<\alpha} F_{\beta}}$ and let

$$
F_{\alpha}=\overline{\bigcup\left\{U_{x}: x \in \text { some } F_{\beta}, \beta<\alpha\right\} \cup U_{y}} .
$$

If $\overline{\bigcup_{\beta<\alpha} F_{\beta}}=X$, let $F_{\alpha}=X$. For $\alpha$ limit with $\operatorname{cf}(\alpha)>\omega$, let $F_{\alpha}=\bigcup_{\beta<\alpha} F_{\beta}$. For such $\alpha, F_{\alpha}$ is then closed by first countability. By construction, for such $\alpha$ 's $F_{\alpha}$ is open as well. Since $X$ is first countable, and compact first 
countable $T_{2}$ spaces have cardinality $\leq 2^{\aleph_{0}}$, it suffices to prove that our construction cannot continue up to say $\mathfrak{c}^{3}+1$. If it did, by just looking at indices of cofinality $\mathfrak{c}$, we would get a strictly increasing $\left(\mathfrak{c}^{2}+1\right)$-sequence of clopen sets. $\mathfrak{c}^{2}+1$ is definable and of size $\mathfrak{c}$ and hence is in $M$, so again by elementarity, there is an $F \in M, F: \mathfrak{c}^{2}+1 \rightarrow \mathcal{T}$, such that for $\alpha<$ $\beta<\mathfrak{c}^{2}+1, F(\alpha) \subsetneq F(\beta)$ and $X-F(\alpha) \in \mathcal{T}$. For each $\alpha<\beta<\mathfrak{c}^{2}+1$, by elementarity again, $F(\alpha) \cap M \subsetneq F(\beta) \cap M$. Thus the $F(\alpha) \cap M$ 's form a strictly increasing sequence of clopen subsets of $X_{M}$. For $\alpha \in \mathfrak{c}^{2}+1$, pick $f(\alpha) \in(F(\alpha+1) \cap M)-(F(\alpha) \cap M)$. Then $\{f(\alpha)\}_{\alpha \in \mathfrak{c}^{2}+1}$ is a free sequence in $X_{M}$, contradicting our hypothesis. Thus Theorem 20 is established.

Now for some more corollaries:

Corollary 24. Suppose $X_{M}$ is a $T_{2}$, locally hereditarily Lindelöf, perfect pre-image of $\omega_{1}$ that contains a point with no countable neighborhood. Then $X=X_{M}$.

Corollary 25. Suppose $X_{M}$ is homeomorphic to the disjoint union of $\omega_{1}$ and $\mathbb{R}$. Then $X=X_{M}$.

Proof. The second one is immediate; for the first, observe that a perfect pre-image of a (locally) compact space is (locally) compact. Then $X_{M}$ is first countable and so has no free sequence of length $>\mathfrak{c}^{2}$.

The cardinal arithmetic hypothesis in Theorem 19(b) is necessary. A proof similar to that for Theorem 20 yields the following:

TheOREM 26. Suppose $2^{\aleph_{0}}>\aleph_{n}$ and $X_{M}$ is locally compact $T_{2}$, locally hereditarily Lindelöf, contains a point with no countable neighborhood, and is the union of $\aleph_{n}$ compact sets. Then $X=X_{M}$.

Note that the "locally hereditarily Lindelöf" condition is essential in these results. Otherwise, one could replace each point in $\omega_{1}$ by the onepoint compactification of the disjoint sum of $\left(2^{\aleph_{0}}\right)^{+}$copies of $[0,1]$. Take a countably closed elementary submodel $M$ of size $2^{\aleph_{0}}$ containing this space $X$. Then $X_{M}$ will be like $X$, except with only $2^{\aleph_{0}}$ copies of $[0,1]$.

Theorem 16(b) shows that the condition that $X_{M}$ include a point with no countable neighborhood is essential if we want ZFC results.

I would like to thank the referee for numerous useful suggestions, including Theorem 19(b). Among other things, s/he noted that if one is mainly interested in Theorem 20 and its corollaries, there is an easier way to obtain $M \supseteq[0,1]$. Lemma 9 is difficult because it assumes only Čech-completeness rather than local compactness. With local compactness, we have:

Lemma 27. If $X_{M}$ includes an open set with uncountable compact first countable closure, then $M \supseteq[0,1]$. 
Proof. If there is such an open set $U$, claim there is one which is of form $W \cap M, W \in M, W$ open in $X$. To see this, first claim each point in $\bar{U}$ is contained in an open set with compact closure, and hence a basic open set with compact closure. For if $p \in \bar{U}-U$, take an open $V$ containing $p$. Then $p \in \overline{U \cap V}$. If each such basic open set had countable closure, by compactness $\bar{U}$ would be countable, contradiction. Let therefore $U=$ $W \cap M, W \in M, W$ open in $X$. Then $\bar{W} \in M$. Note $\bar{U}=(\bar{W})_{M}$; that $(\bar{W})_{M}=\bar{W} \cap M \subseteq \bar{U}$ is clear; if $x \in \bar{U}$ were not in $\bar{W}$, there would be a $V$ open in $X$ and containing $x$ such that $V \cap U=0$. Take such a $V \in M$. Then $V \cap M \cap U=0$, contradiction. Since $(\bar{W})_{M}$ is compact, so is $\bar{W}$ [Ju] and so by Lemma 6 there is a perfect map from $\bar{W}$ onto $[0,1]$. But then $(\bar{W})_{M}$ maps onto $[0,1]_{M}=[0,1] \cap M$, whence by compactness, $[0,1] \subseteq M$.

\section{References}

[AL] J. M. Aarts and D. J. Lutzer, Completeness properties designed for recognizing Baire spaces, Dissertationes Math. 116 (1974).

[A] A. V. Arkhangel'skiı̌, On bicompacta hereditarily satisfying Suslin's condition. Tightness and free sequences, Soviet Math. Dokl. 12 (1971), 1253-1257.

[E] R. Engelking, General Topology, Heldermann, Berlin, 1989.

[F] Z. Frolík, Generalizations of the $G_{\delta}$-property of complete metric spaces, Czechoslovak Math. J. 10 (1960), 359-379.

[H] R. Hodel, Cardinal functions I, in: Handbook of Set-Theoretic Topology, NorthHolland, Amsterdam, 1984, 1-61.

$\left[\mathrm{J}_{1}\right] \quad$ I. Juhász, Cardinal Functions in Topology, Math. Centrum, Amsterdam, 1971.

$\left[\mathrm{J}_{2}\right] \quad-$, Cardinal Functions in Topology - Ten Years Later, Math. Centrum, Amsterdam, 1980.

[JNW] I. Juhász, Zs. Nagy and W. Weiss, On countably compact, locally countable spaces, Period. Math. Hungar. 10 (1979), 193-206.

[Ju] L. R. Junqueira, Upwards preservation by elementary submodels, Topology Proc. 25 (2000), 225-249.

[JT] L. R. Junqueira and F. D. Tall, The topology of elementary submodels, Topology Appl. 82 (1998), 239-266.

[JW] W. Just and M. Weese, Discovering Modern Set Theory, II, Amer. Math. Soc., Providence, 1997.

[K] A. Kanamori, The Higher Infinite, Springer, Berlin, 1994.

[Ke] A. S. Kechris, Classical Descriptive Set Theory, Springer, New York, 1994.

[Ku] K. Kunen, Set Theory, North-Holland, Amsterdam, 1984.

[KT] K. Kunen and F. D. Tall, The real line in elementary submodels of set theory, J. Symbolic Logic 65 (2000), 683-691.

[P] R. G. A. Prado, Applications of reflection to topology, Ph.D. thesis, Univ. of Toronto, 1999.

[PT] R. G. A. Prado and F. D. Tall, Characterizing $\omega_{1}$ and the long line by their topological elementary reflections, Israel J. Math. 127 (2002), 81-91.

[T] F. D. Tall, If it looks and smells like the reals..., Fund. Math. 163 (2000), 1-11. 
[W] P. D. Welch, On possible non-homeomorphic substructures of the real line, Proc. Amer. Math. Soc. 130 (2002), 2771-2775.

Department of Mathematics

University of Toronto

Toronto, Ontario M5S 3G3, Canada

E-mail: tall@math.utoronto.ca

Received 22 November 2001;

in revised form 1 September 2002 